\title{
Treatment of social phobia through pure self-help and therapist-augmented self-help
}

\author{
RONALD M. RAPEE, MAREE J. ABBOTT, ANDREW J. BAILLIE \\ and JONATHAN E. GASTON
}

\section{Background Self-help for social phobia has not received controlled empirical evaluation.}

\begin{abstract}
Aims To evaluate the efficacy of pure self-help through written materials for severe social phobia and self-help augmented by five group sessions with a therapist. These conditions were compared with a waiting-list control and standard, therapist-led group therapy.
\end{abstract}

Method Participants with severe generalised social phobia $(n=224)$ were randomised to one of four conditions.

Assessment included diagnoses, symptoms and life interference at pretreatment, 12 weeks and at 24 weeks.

Results A larger percentage of patients no longer had a diagnosis of social phobia at post-intervention in the pure self-help group than in the waiting-list group, although this percentage decreased slightly over the next 3 months. Symptoms of social anxiety and life interference did not differ significantly between these groups. Augmented self-help was better than waiting list on all measures and did not differ significantly from group treatment.

Conclusions Self-help augmented by therapist assistance shows promise as a less resource-intensive method for the management of social phobia. Pure selfhelp shows limited efficacy for this disorder.

Declaration of interest R.M.R. authored the book used for bibliotherapy and receives royalties.
Although treatments for social phobia (social anxiety disorder) have shown good efficacy (Gould, et al, 1997; Fedoroff \& Taylor, 2001), traditional models of treatment delivery are associated with several limitations. These include their cost, the limited availability of mental health workers and most especially the small percentage of people with this disorder who seek traditional treatment (Meltzer et al, 2000; Issakidis \& Andrews, 2002). Self-help and minimal therapist treatments provide a possible alternative to traditional therapy models. Bibliotherapeutic interventions have been applied with success to a wide range of difficulties (Scogin et al, 1990; Marrs, 1995), including anxiety disorders (Newman et al, 2003; Barlow et al, 2005). In contrast to research into other anxiety disorders, there has been little investigation into bibliotherapy for social phobia (e.g. Newman et al, 2003). The few studies that have been conducted have all included some degree of researcher involvement, hence limiting the conclusions that can be drawn about 'pure' self-help. Our study was designed to determine the value of two forms of self-help through the use of bibliotherapeutic materials in the reduction of social phobia: pure bibliotherapy that involved almost no contact with the researchers, and therapist-augmented bibliotherapy in which printed material was supplemented with five group sessions conducted by a therapist. Benchmarks for these conditions were provided by comparison with a no-treatment waiting list and standard ten-session group therapy conducted by a therapist.

\section{METHOD}

\section{Participants}

Participants for the study were 224 individuals meeting DSM-IV criteria (American Psychiatric Association, 1994) for social phobia, randomly allocated to one of four treatment conditions: standard group treatment, 'pure' self-help, self-help augmented with minimal therapist assistance, and waiting list. Participants were included if they were aged 20-65 years, met criteria for social phobia as their main (or most interfering) disorder, and had sufficient English and education to read a tabloid newspaper in English. In order to maximise external validity, exclusions were kept to a minimum. The only planned exclusions were problems requiring immediate attention such as clear suicidal intent, severe substance misuse or dependence, or florid psychosis, assessed during the structured interviews. Concurrent pharmacotherapy or psychotherapy was allowed as long as dosages had been consistent for 3 months and there was no plan to change. No participant was in concurrent psychotherapy. However, $6.8 \%$ were taking benzodiazepines or other anxiolytics, $21.2 \%$ were taking selective serotonin reuptake inhibitors or other antidepressants and 9.9\% were taking other prescription medications.

Diagnoses of Axis I disorders were made by graduate students in clinical psychology using a structured clinical interview, the Anxiety Disorders Interview Schedule for DSM-IV (ADIS-IV; Di Nardo et al, 1994). Data from our laboratory using this interview and including a proportion of the current sample have indicated a moderate to strong interrater reliability for diagnoses of anxiety and mood disorders, including a very high reliability for a diagnosis of social phobia ( $\kappa=0.89$ ). In addition, the avoidant personality disorder questions from the ICD-10 International Personality Disorder Examination (Loranger et al, 1997) were also asked of all participants. Interrater reliability for a diagnosis of avoidant personality disorder was moderate $(\kappa=0.65)$. Among the current sample, $95.7 \%$ met criteria for the generalised subtype of social phobia and $55.8 \%$ met criteria for a diagnosis of avoidant personality disorder. As would be expected in such a severely affected sample, Axis I comorbidity was also high: $42.9 \%$ met criteria for an additional anxiety disorder, $33.9 \%$ met criteria for an additional mood disorder and $4.0 \%$ met criteria for an additional substance use or alcohol disorder. The mean age of the sample was 35.5 years (s.d.=11.0) and $50.4 \%$ were female.

\section{Measures}

Participants were assessed with the following measures at a pre-treatment interview 
and 12 weeks later. Participants in active treatment were also followed up 12 weeks after that (24 weeks after the initial assessment).

\section{Social Interaction Anxiety Scale and Social Phobia Scale}

The Social Interaction Anxiety Scale (SIAS) and the Social Phobia Scale (SPS) (Mattick \& Clarke, 1998) are companion scales that assess the main fears and avoidance of social phobia, focusing respectively on interaction fears and more specific performance-based fears. They have excellent psychometric properties (Peters, 2000).

\section{Brief Fear of Negative Evaluation scale}

The Brief Fear of Negative Evaluation scale (BFNE; Leary, 1983) assesses the cognitive aspects relevant to social phobia, especially those related to negative evaluation. Psychometric properties are sound and it has shown stronger validity that the previous Fear of Negative Evaluation scale (Rodebaugh et al, 2004)

\section{Albany Panic and Phobia Questionnaire}

The Albany Panic and Phobia Questionnaire social phobia sub-scale (APPQ-S; Rapee et al, 1994) is a brief set of items designed to tap social fears that are relatively distinct from overlap with agoraphobic fears. Later examination has shown consistent factor structure, solid reliability and clear concurrent validity (Brown et al, 2005).

\section{Self Consciousness Scale}

The Self Consciousness Scale social anxiety sub-scale (SCS-A; Fenigstein et al, 1975) is a six-item scale containing items tapping a variety of broader aspects of shyness and social reticence. It has shown solid psychometric properties in a number of translations and has been widely used in the social anxiety literature.

\section{Life Interference Scale}

To provide a measure of the life impact of individuals' social fears, six Likert scales (scored 0-8) asked respondents to indicate the impact of their fears on various components of their life including work, family life and leisure activities. The scales were summed to provide a total interference rating from 0 (no interference) to 48 (maximum interference). Previous analysis in our centre has shown that the six items show excellent internal consistency $(\alpha=0.90)$ and the total correlates significantly with the 12-item Short-Form Health Survey (SF-12; Ware et al, 1996) mental component sub-scale $(r=0.56)$.

\section{Treatment conditions}

\section{Standard group treatment}

Standard treatment was included to represent the 'gold standard' treatment effect. Treatment was conducted in groups of approximately six participants with two graduate psychology student therapists who received minimal supervision from an experienced clinical psychologist. Therapy extended for ten $2 \mathrm{~h}$ sessions across 12 weeks. Treatment was manualised, based on principles and components described in a book by Rapee \& Sanderson (1998). Components included those typically found in empirically validated treatments for social phobia including cognitive restructuring of negative evaluation beliefs, exposure to feared social situations, realistic feedback of social performance, and attention training. Participants engaged in home exercises and received various handouts as relevant.

\section{'Pure' self-help}

Participants were given a copy of the book Overcoming Shyness and Social Phobia: A Step by Step Guide (Rapee, 1998) and told to read it and work their way through the exercises described in the book. The strategies outlined paralleled those in the standard group treatment, and practice sheets and exercises formed part of the book. In order to encourage a reasonable rate of progress, participants were given a cover letter with the book welcoming them to the programme and providing a suggested rate of progress in order to complete it in 12 weeks. They were told that post-treatment assessment would occur at 12 weeks and thereafter they would have no additional contact with the researchers. This condition was designed to simulate conditions under which a person might obtain written materials without professional assistance (for example, buying the book in a shop or being given a manual while waiting for treatment).

\section{Self-help augmented by therapist assistance}

Participants in the augmented self-help condition were given a copy of the same book as those in the pure self-help group and told to read and practise the exercises described. They also met in groups of five to seven participants with a therapist (a graduate psychology student) on five occasions across the 12 weeks. Each group session ran for $2 \mathrm{~h}$; thus, the total therapist time was exactly half of that in the standard group therapy programme. The same therapists participated in this condition and in the standard treatment condition. The aims of the group sessions were to problem-solve application of the principles described in the book to the personal context of each participant and to provide motivation and encouragement to apply these principles.

\section{Waiting list}

Participants on the waiting list were told that they had been randomly allocated to receive no treatment for 12 weeks. At the end of the 12-week period they were offered our best available treatment.

\section{Procedure}

Potential participants contacted the Macquarie University Anxiety Research Unit through the usual referral sources, including general practitioners, mental health professionals, occasional media coverage and word of mouth. These volunteers were screened by telephone and those who appeared to have social anxiety-related difficulties were invited to attend for a structured interview. Those who met inclusion criteria were randomly allocated to one of the four conditions. Randomisation was done using a pre-assigned random number generator in blocks of eight to allow for group delivery. Participants in the pure self-help group were given a copy of the book and the cover letter and were then simply contacted again after 12 weeks for a second assessment. Participants in the augmented self-help group were given a copy of the book and a schedule of group meetings. Participants in the standard treatment group were simply given a schedule of meeting times. The procedures were approved by the Macquarie University human research ethics committee.

\section{Statistical analysis}

Primary outcomes for this trial were a reduction in clinical diagnoses of social phobia as assessed by the ADIS-IV, reduction in a composite of social phobia symptom 
measures, and reductions in self-rated life interference.

Following earlier research (Clark et al, 1994), several related symptom measures were grouped together and combined into a standardised composite to reduce the number of statistical tests performed and hence the type 1 error rate. A composite score was produced to represent total social phobia symptom severity. This comprised scores on the clinician-rated severity of social phobia derived from the ADIS-IV, the SPS and SIAS, the social phobia subscale of the APPQ, the social anxiety subscale of the SCS and the BFNE. Scores on each scale were standardised across all groups on all measurement occasions before being summed and re-standardised to give a mean for all groups and all measurement occasions of 0 and a standard deviation of 1 .

The proportions of participants whose phobia was in remission at the posttreatment assessments and 3-month followup in each treatment condition were compared using Fisher's exact test. Differences between treatments in change in the standardised social phobia symptom composite and rating of life interference were examined using mixed models containing random intercept and random slope terms as well as fixed effects for treatment received (Gibbons et al, 1993). All analyses were conducted using the Statistical Package for the Social Sciences version 13.0.1 for Windows. Confidence intervals for the number needed to treat were calculated following Altman (1998).

\section{Missing data}

The number of participants who provided no data at post-treatment and at 3-month follow-up is shown in Fig. 1. The last value carried forward strategy was used to substitute missing data if data were not available at the 3-month follow-up or at both post-treatment and 3-month followup. Interpolation was used if post-treatment data only were not available. As a precaution against biasing effects of these methods of handling missing data, analyses were conducted with and without missing data substituted. Analyses with missing data substituted are equivalent to intentto-treat analyses. However, analyses without missing data substituted are not equivalent to so-called 'completer' analyses. In most clinical trials completer analyses include only those participants who receive a sufficient 'dose' of treatment (e.g. attend enough treatment sessions); however, this cannot be determined in participants undergoing self-help. Therefore analyses without missing data substitution may include some participants who did not implement any of the self-help, even though they returned data. There was no significant difference in the pre-treatment social phobia symptom composite score between those who provided post-treatment and follow-up data and those who did not $\left(t_{(222)}=-1.135, P>0.05\right)$.

\section{RESULTS}

Demographic data on the sample broken down across the four allocated groups are presented in Table 1.

\section{Diagnosis-free status}

The number and percentage of participants from each treatment condition who no longer met criteria for a diagnosis of social phobia at the post-treatment and follow-up assessments are shown in Table 2. At posttreatment assessment, participants who received active treatments showed significantly greater diagnosis-free rates (group treatment $22 \%, n=13$; augmented self-help $19 \%, n=11$; pure self-help $20 \%, n=11$ ) than the waiting-list group $(6 \%, n=3$; Fisher's exact test $P<0.008)$. There was no significant difference in diagnosis-free rates at post-treatment assessment between those who received some form of group therapy (augmented self-help 19\%, $n=11$; group treatment $22 \%, n=13$ ), and those who received pure self-help $(20 \%, n=11$; Fisher's exact test $P=0.522$ ).

Table I Characteristics of the four groups of participants

\begin{tabular}{lcccc}
\hline & Waitinglist & $\begin{array}{c}\text { Pure } \\
\text { self-help }\end{array}$ & $\begin{array}{c}\text { Self-help } \\
\text { augmented with } \\
\text { therapist } \\
\text { assistance }\end{array}$ & $\begin{array}{c}\text { Group } \\
\text { treatment }\end{array}$ \\
\hline Age, years: mean (s.d.) & $36.2(11.6)$ & $36.5(10.1)$ & $34.8(10.1)$ & $34.8(12.1)$ \\
Female, $n$ (\%) & $23(44)$ & $33(59)$ & $27(47)$ & $30(51)$ \\
Married, $n$ (\%) & $15(29)$ & $26(46)$ & $21(37)$ & $12(20)$ \\
Post high-school education, $n$ (\%) & $32(63)$ & $42(75)$ & $36(64)$ & $43(74)$ \\
Avoidant personality disorder, $n$ (\%) & $31(60)$ & $23(41)$ & $35(61)$ & $36(61)$ \\
Any secondary Axis I diagnosis, $n(\%)$ & $33(66)$ & $37(66)$ & $37(65)$ & $38(64)$ \\
Prescription medication, $n$ (\%) & $20(39)$ & $13(23)$ & $17(30)$ & $20(34)$ \\
\hline
\end{tabular}

At 3-month follow-up there were significantly more participants who no longer met ADIS-IV diagnostic criteria for social phobia in the group treatment and augmented self-help conditions $(22 \%, n=13 ; 26 \%$, $n=15$, respectively) compared with the selfhelp condition $(11 \%, n=6$; Fisher's exact test $P<0.05$ ).

The number needed to treat comparing pure self-help with augmented self-help is 7 $(1 /(0.107-0.263)=6.4)$ with a $95 \%$ confidence interval of 3.4 to 62.8 . This indicates that seven patients with social phobia need to be treated with bibliotherapy augmented by face-to-face group interventions before one additional patient achieves a reduction in social phobia over and above that achieved from bibliotherapy alone.

\section{Change in composite outcome measures}

Changes in the mean standardised composite of social phobia symptom measures and standardised life interference ratings are shown in Table 3, as are the changes from pre-treatment to 24-week follow-up expressed as standardised mean difference effect sizes. In order to facilitate comparison of our data with other research, an additional table has been included that lists means and standard deviations for several of the main outcome measures (Table 4). To maintain a reasonable type 1 error rate, these individual scores were not subjected to independent statistical analyses - they are for descriptive purposes only.

Hierarchical linear or mixed models containing random intercept and slope parameters were fitted to the standardised composite social phobia symptom measures, and to standardised ratings of life 
Table 2 Participants without a clinical diagnosis of social phobia at post-treatment and 3 month follow-up assessments

\begin{tabular}{lccc}
\hline & & \multicolumn{2}{c}{ No social phobia } \\
\cline { 3 - 4 } & $n$ & $\begin{array}{c}\text { Post-treatment } \\
n(\%)\end{array}$ & $\begin{array}{c}\text { 3-month follow-up' } \\
n(\%)\end{array}$ \\
\hline Waiting list & & $3(6 \%)$ & Not Assessed \\
Pure self-help & 52 & II (20\%) & $6(11 \%)$ \\
Self-help augmented with therapist assistance & 56 & II (19\%) & $15(26 \%)$ \\
Group treatment & 57 & $13(22 \%)$ & $13(22 \%)$ \\
\hline
\end{tabular}

I. Those who did not provide follow-up data are assumed to still have the condition. interference due to social anxiety. The random intercept parameter allows for individuals differing within groups on their level of severity, whereas the random slope parameter allows for within-group variance in the rate of change over time. Models with an autoregressive covariance structure for the random slope effect were attempted but did not converge, so results from models with diagonal covariance structure for the random slope are described. Because of a trend toward differences between groups in the pre-treatment diagnosis of avoidant personality disorder $\left(\chi^{2}=6.196\right.$, d.f. $=3, P=0.102)$, pre-treatment clinicianrated severity of avoidant personality disorder was included as a covariate. The best-fitting model containing random intercept, slope, pre-treatment avoidant personality disorder and treatment effects gave a $-2 \log$ likelihood of 1104.298. (Because around $30 \%$ of the sample were taking medication, we tested models that contained medication use as a covariate; there was no improvement in model fit, and hence medication use was not included in the analyses.)

There was a significant group by time interaction on the social phobia composite $\left(F_{(4,216.9)}=16.131, P<0.001\right)$, so planned follow-up tests were conducted to examine specific differences between groups. There was a trend toward differences between the pure self-help and waiting-list interventions post-treatment $\quad\left(t_{(246.443)}=1.69\right.$, $P=0.093)$, and significant differences

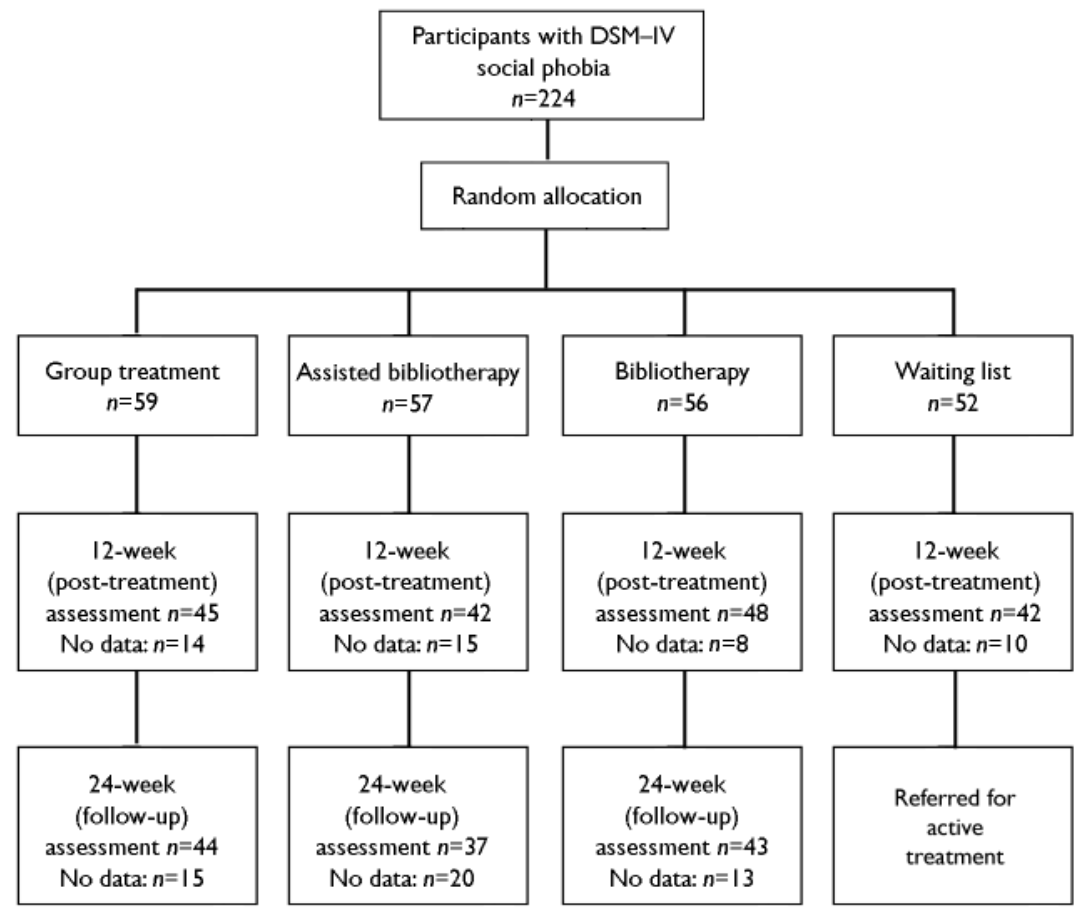

Fig. I Study profile. between waiting list and augmented selfhelp $\left(t_{(247.133)}=4.457, P<0.001\right)$ and group treatment $\left(t_{(247.060)}=4.131, P<0.001\right)$ also both at post treatment.

At the 24-week follow-up assessment, augmented self-help and group treatment resulted in significantly lower levels of the standardised social phobia composite than the pure self-help condition (augmented $v$. pure self-help $t_{(254.695)}=-3.582, P<0.001$; group treatment $v$. pure self-help $t_{(254.120)}$ $=-3.447, P<0.001)$. There was no significant difference between the augmented self-help and group treatment for this measure at the 24-week assessment $\left(t_{(254.900)}=0.137\right.$, NS). The same pattern of results was observed when missing data were excluded.

Similar results were observed for ratings of the extent to which social anxiety interfered with a range of activities (total score on the Life Interference Scale). A mixed model containing random intercept and slope terms and including baseline clinician-rated severity of avoidant personality disorder as a covariate was the best fit to the data and gave a $-2 \log$ likelihood of 1368.75 . There was a significant group by time interaction $\left(F_{(4,258.892)}=7.4398\right.$, $P<0.001)$ indicating that participants in the four conditions changed at significantly different rates. At the post-treatment assessment, augmented self-help and group treatment led to significantly lower ratings of life interference than the waiting-list control (augmented self-help $v$. waiting list $t_{(234.272)}=-2.577, P<0.01$; group treatment $v$. waiting list, $t_{(234.243)}=-2.41$, $P<0.02$ ) while there was no significant difference between the pure self-help and waiting list groups $\left(t_{(233.998)}=-0.716, \mathrm{NS}\right)$. At 24-week follow-up both augmented self-help and group treatment led to significantly less life interference compared with the pure self-help condition (augmented $v$. pure self-help, $t_{(249.894)}=-2.514, P<0.02$; group treatment $v$. pure self-help $\left.t_{(249.671)}=-2.236, P<0.05\right)$ with no significant difference between the two interventions involving group therapy $\left(t_{(249.972)}=\right.$ 0.294 , NS). Again results were consistent when missing data were not substituted.

\section{Mediation of change in bibliotherapy}

Participants in the two conditions that involved use of the self-help book differed significantly in the number of chapters they reported reading: pure self-help 4.11 , 
Table 3 Continuous outcome measure scores over time

\begin{tabular}{|c|c|c|c|c|c|c|c|c|}
\hline & \multicolumn{2}{|c|}{ Pre-treatment } & \multicolumn{2}{|c|}{ Post-treatment } & \multicolumn{2}{|c|}{ 3-month follow-up } & \multicolumn{2}{|c|}{ Effect size } \\
\hline & Mean & $(\text { s.e.m. })^{\prime}$ & Mean & (s.e.m.) & Mean & (s.e.m.) & Pre to post ${ }^{2}$ & Pre to follow-up ${ }^{3}$ \\
\hline \multicolumn{9}{|l|}{ Waiting list $(n=52)$} \\
\hline Standardised life interference & 0.305 & $(0.148)$ & 0.226 & $(0.126)$ & & & 0.074 & \\
\hline Standardised social phobia symptom composite & 0.445 & $(0.11 \mathrm{I})$ & 0.285 & $(0.100)$ & & & 0.200 & \\
\hline \multicolumn{9}{|l|}{ Pure self help $(n=56)$} \\
\hline Standardised life interference & 0.397 & $(0.143)$ & 0.099 & $(0.123)$ & 0.069 & $(0.130)$ & 0.278 & 0.307 \\
\hline Standardised social phobia symptom composite & 0.401 & $(0.107)$ & 0.050 & $(0.097)$ & 0.045 & $(0.101)$ & 0.438 & 0.444 \\
\hline \multicolumn{9}{|l|}{ Augmented self-help $(n=57)$} \\
\hline Standardised life interference & 0.464 & $(0.141)$ & -0.225 & $(0.121)$ & -0.391 & $(0.128)$ & 0.647 & 0.803 \\
\hline Standardised social phobia symptom composite & 0.397 & $(0.106)$ & -0.330 & $(0.095)$ & -0.459 & $(0.099)$ & 0.908 & 1.075 \\
\hline \multicolumn{9}{|l|}{ Group treatment $(n=59)$} \\
\hline Standardised life interference & 0.531 & $(0.139)$ & -0.191 & $(0.119)$ & -0.338 & $(0.126)$ & 0.676 & 0.815 \\
\hline Standardised social phobia symptom composite & 0.443 & $(0.104)$ & -0.281 & $(0.094)$ & $-0.44 I$ & $(0.098)$ & 0.906 & 1.105 \\
\hline
\end{tabular}

I. Means are estimated marginal means with the level of the clinician-rated severity of avoidant personality disorder set at the overall pre-treatment mean (2.783) and missing data substituted by the last observed value or the interpolation of adjacent values (described in more detail in the method section).

2. Difference between the pre-treatment and post-treatment means divided by the pre-treatment standard deviation (the pre-treatment standard deviation was used because missing data substitution might have reduced the variance of later observations and thus unduly inflated effect size estimates).

3. Difference between the pre-treatment and follow-up means divided by the pre-treatment standard deviation.

(s.d.=2.95); augmented self-help 7.48 (s.d.=2.08, $t(59)=3.37, P<0.001)$. The number of chapters read was significantly related to the overall level of the social phobia symptom composite $\left(F_{(13,95.099)}=1.858\right.$, $P<0.05)$, indicating that the more severe the social anxiety in general the greater the number of chapters read. Number of chapters read was also significantly related to the rate of change in the social phobia symptom composite $\left(F_{(26,124.804)}=1.677\right.$, $P<0.05)$, so that reading more chapters was associated with a significantly greater improvement. However, the interaction between time, number of chapters read and treatment condition was not significant $\left(F_{(8,119.649)}=1.372, \mathrm{NS}\right)$, indicating that the number of chapters read did not explain

Table 4 Main social anxiety symptom measure scores

\begin{tabular}{|c|c|c|c|c|c|c|c|c|}
\hline & \multicolumn{2}{|c|}{ Pre-treatment } & \multicolumn{2}{|c|}{ Post-treatment } & \multicolumn{2}{|c|}{ 3-month follow-up } & \multicolumn{2}{|c|}{ Effect size } \\
\hline & Mean & (s.d. $)^{\prime}$ & Mean & (s.d.) & Mean & (s.d.) & Pre to post ${ }^{2}$ & Pre to follow-up ${ }^{3}$ \\
\hline \multicolumn{9}{|c|}{ Waiting list $(n=52)$} \\
\hline SIAS & 54.686 & (13.679) & 54.417 & $(13.508)$ & & & 0.020 & \\
\hline SPS & 37.529 & $(15.142)$ & 35.337 & $(15.830)$ & & & 0.145 & \\
\hline BFNE & 49.823 & (7.543) & 48.843 & (7.528) & & & 0.130 & \\
\hline \multicolumn{9}{|c|}{ Pure self-help $(n=56)$} \\
\hline SIAS & 51.125 & $(13.339)$ & 46.057 & $(I 7.42 I)$ & 47.143 & $(15.953)$ & 0.380 & 0.299 \\
\hline SPS & 36.429 & $(17.173)$ & 30.962 & (17.679) & 31.464 & $(17.676)$ & 0.318 & 0.289 \\
\hline BFNE & 50.125 & (8.594) & 45.755 & $(9.589)$ & 47.679 & $(9.280)$ & 0.509 & 0.285 \\
\hline \multicolumn{9}{|c|}{ Augmented self-help $(n=57)$} \\
\hline SIAS & 55.789 & $(13.227)$ & 43.236 & $(16.650)$ & 41.596 & $(16.387)$ & 0.949 & 1.073 \\
\hline SPS & 34.456 & $(16.250)$ & 24.429 & $(16.867)$ & 23.491 & $(17.068)$ & 0.617 & 0.675 \\
\hline BFNE & 51.125 & $(6.735)$ & 44.625 & $(9.316)$ & 43.053 & $(9.420)$ & 0.965 & 1.199 \\
\hline \multicolumn{9}{|c|}{ Group treatment $(n=59)$} \\
\hline SIAS & 54.237 & $(12.343)$ & 44.333 & $(I 5.047)$ & 42.203 & $(15.883)$ & 0.802 & 0.975 \\
\hline SPS & 38.475 & $(14.536)$ & 28.630 & $(15.010)$ & 26.034 & $(I 5.375)$ & 0.677 & 0.856 \\
\hline BFNE & 51.254 & $(6.997)$ & 45.000 & $(8.795)$ & 43.254 & $(9.325)$ & 0.894 & $\mathrm{I} .143$ \\
\hline
\end{tabular}

BFNE, Brief Fear of Negative Evaluation; SIAS, Social Interaction Anxiety Scale; SPS, Social Phobia Scale.

I. Means are calculated with missing data substituted by the last observed value or the interpolation of adjacent values (described in more detail in the method section).

2. Difference between the pre-treatment and post-treatment means divided by the pre-treatment standard deviation (the pre-treatment standard deviation was used because missing data substitution might have reduced the variance of later observations and thus unduly inflated effect size estimates).

3. Difference between the pre-treatment and follow-up means divided by the pre-treatment standard deviation. 
the differences in the rate of change between the book only and book plus group interventions.

\section{DISCUSSION}

\section{Value of pure self-help}

The major question addressed in this study was whether marked reductions in social phobia could be achieved through self-help delivered in the form of printed material. The results provided mixed support for the value of bibliotherapy in reducing both social fears and the degree of life interference caused by social anxiety. Specifically, the extent of the reductions was markedly influenced by the method of delivering bibliotherapy.

When bibliotherapy was delivered in a 'pure' form - that is, with no significant involvement from a therapist - results were relatively modest. A reasonable proportion of patients no longer met diagnostic criteria for social phobia using pure self-help, although this proportion appeared to be declining by the follow-up point. Changes in symptoms showed a trend to be greater than those of the waiting-list control alone and were maintained reasonably over time, but reductions in life interference were not significantly greater than in the waiting-list group. Hence as a clinical intervention, pure bibliotherapy appears to show limited value for social phobia. However, the modest indications of efficacy (e.g. the moderate effect size change in life interference) suggest that pure bibliotherapy could have a role in population-level interventions or in provision of help to groups who might not have access to extensive mental health services. However, such a suggestion would require more thorough investigation including sample sizes sufficient to detect the small effect sizes that might still have benefits across an entire population.

From a theoretical perspective the modest efficacy of pure self-help for social phobia stands in interesting contrast to the stronger effects shown with many other disorders (Scogin et al, 1990; Marrs, 1995; Newman et al, 2003; Barlow et al, 2005). Social phobia is one of the most chronic of the anxiety disorders (Bruce et al, 2005) and has marked personality-like characteristics (Rapee \& Spence, 2004). Hence self-help may be far more difficult to conceptualise and implement for this ego-syntonic condition than for disorders that involve more overt shifts from normal functioning. Our sample was also especially severely phobic and contained a large proportion of people with avoidant personality disorder. It is possible that individuals with more circumscribed forms of social phobia might be more amenable to self-help, although interestingly our data indicated that it was the more severely affected individuals who read more chapters of the book. Finally, the underlying fears in social phobia (e.g. 'if I make a mistake people will think badly of me') are typically far less veridical and hence more open to biases in interpretation than many of the concerns in other disorders (e.g. 'riding on a bus will lead to a heart attack'). This feature may make social phobia less amenable than other disorders to pure self-help. Although the current study provided one of the most valid tests of pure self-help, it is not possible to test a true model of self-help as it would be used in the real world. Specifically, self-help in our study differed from real-world use through the inclusion of pre-treatment assessment and contact, a contact letter, the 'structure' of a research trial, and post-treatment assessments. These inclusions might have led to overestimation of the efficacy of pure self-help.

\section{Augmented self-help}

In contrast to pure self-help, augmentation of self-help with five therapist-led group sessions resulted in marked improvements in symptoms of social phobia and life interference that were as great as those produced by standard group treatment. The lack of a five-session, therapist-only condition does not allow complete conclusions to be drawn about the role of written materials. Although unlikely, it is possible that five group sessions with a therapist might have resulted in equivalent benefit to the augmented bibliotherapy. Nevertheless, this method may provide a template for a highly resource-effective method of treatment delivery. The effect size change in social phobia symptoms produced by augmented self-help (1.08) was larger than the typical effects of cognitive-behavioural therapy shown in meta-analyses (around 0.8) (Fedoroff \& Taylor, 2001). Interestingly, a recent treatment for social phobia using internet-delivered self-help combined with some therapist input and in vivo exposure demonstrated an effect size of 0.87 (Andersson et al, 2006). Although treatment based on more recent models of social phobia has shown larger effects, this is accompanied by a markedly increased cost (e.g. Clark et al, 2003). Hence we can begin to flesh out the range of options available to mental health services. At one extreme, expert therapists treating individual patients under detailed supervision can produce extremely efficacious results at a higher cost and limited accessibility. At the other extreme, simple provision of printed materials can produce small changes at extremely low cost and broad accessibility. Augmentation of printed materials with a few therapist-led sessions provides one mid-point alternative. Future research needs to explore further alternatives that might provide the best balance between efficacy and resource use. As an example, John Walker and colleagues (personal communication) have shown good effects from augmenting bibliotherapy with group sessions led by lay facilitators.

\section{Mechanisms of change}

Further improvements in the efficacy of bibliotherapy could come from research into mediators of change. The results of our study showed that the amount of reading was positively related to outcome. Although this is not surprising, it does imply that identifying methods to increase reading of materials might increase the efficacy of bibliotherapy. Surprisingly, although the use of therapist augmentation was associated with a considerably greater amount of reading, this difference did not explain significant variance in the differences between groups. It appears that therapist augmentation of bibliotherapy provides benefits over and above simple motivation to read the materials. Candidate variables could include better interpretation of procedures, training in additional strategies or more positive outcome expectancies. Several other methods of augmentation have shown promise, including return of weekly homework tasks, 'check-in' and reminders through post, telephone, palmtop computers or email. Electronic delivery of self-help is enjoying popularity and may result in some benefits. In many cases internet systems simply consist of written materials in electronic form and will provide no greater benefit than printed materials. However, the use of sophisticated computer programs does allow several interesting features such as individually tailored applications, regular feedback and tracking of progress, and built-in reminders (Griffiths \& Christensen, 2006). 
Research into the efficacy of bibliotherapy would benefit from systematic examination of predictors. Significant predictors should be used both to screen participants who are most likely to benefit (Baillie $\&$ Rapee, 2004) and to inform the development of future modes of delivery.

\section{Implications}

Mental health services around the world are limited in their reach and scope. In addition, a large proportion of people with anxiety disorders including social phobia do not seek help from traditional mental health services (Meltzer et al, 2000; Issakidis \& Andrews, 2002). Many of these people report preferring to deal with difficulties themselves (Issakidis \& Andrews, 2002). For these people in particular, self-help might provide an acceptable alternative to traditional therapy. Advantages of self-help include freeing up mental health professionals to allow them to deal with individuals who do require more intensive intervention (Baillie \& Rapee, 2004) and providing a more easily accessible and less stigmatising alternative for individuals who are unwilling or unable to access traditional services. Thus, continued investigation into the efficacy of self-help methods can have major implications for public health. Several studies have demonstrated the value of self-help for a variety of anxiety disorders. The current data suggest that pure self-help appears to be less efficacious for social phobia than for other anxiety disorders. Nevertheless, the indications shown here for small effects suggest that larger studies with clearer implications for population health would be of value.

\section{REFERENCES}

Altman, D. G. (1998) Confidence intervals for the number needed to treat. BMJ, 317, 1309-1312.

American Psychiatric Association (1994) Diagnostic and Statistical Manual of Mental Disorders (4th edn) (DSM-IV). APA.

Andersson, G., Carlbring, P., Holmstrom, A., et al (2006) Internet-based self-help with therapist feedback and in vivo exposure for social phobia: a randomized controlled trial. Journal of Consulting and Clinical Psychology, 74, 677-686.

Baillie, A. J. \& Rapee, R. M. (2004) Predicting who benefits from psychoeducation and self help for panic attacks. Behaviour Research and Therapy, 42, 513-527.

RONALD M. RAPEE, PhD, Department of Psychology, Macquarie University, Sydney; MAREE J. ABBOTT, PhD, Department of Psychology, University of Sydney; ANDREW J. BAILLIE, MPsychol, PhD, Department of Psychology, Macquarie University, Sydney; JONATHAN E. GASTON, MClinPsych, Macquarie University Anxiety Research Unit, Macquarie University, Sydney, New South Wales, Australia

Correspondence: Dr Ronald M. Rapee, Department of Psychology, Macquarie University, Sydney, NSW 2109, Australia. Email: Ron.Rapee@mq.edu.au

(First received 29 June 2006, final revision 10 April 2007, accepted 14 May 2007)

Barlow, J. H., Ellard, D. R., Hainsworth, J. M., et al (2005) A review of self-management interventions for panic disorders, phobias and obsessive-compulsive disorders. Acta Psychiatrica Scandinavica, III, 272-285.

\section{Brown, T. A., White, K. S. \& Barlow, D. H. (2005)}

A psychometric reanalysis of the Albany Panic and Phobia Questionnaire. Behaviour Research and Therapy, 43. 337-355.

Bruce, S. E., Yonkers, K. A., Otto, M. W., et al (2005) Influence of psychiatric comorbidity on recovery and recurrence in generalized anxiety disorder, social phobia, and panic disorder: a 12-year prospective study. American Journal of Psychiatry, 162, |179-1187.

Clark, D. M., Salkovskis, P. M., Hackmann, A., et al (1994) A comparison of cognitive therapy, applied relaxation and imipramine in the treatment of panic disorder. British Journal of Psychiatry, 164, 759-769.

Clark, D. M., Ehlers, A., McManus, F., et al (2003) Cognitive therapy versus fluoxetine in generalized social phobia: a randomized placebo-controlled trial. Journal of Consulting and Clinical Psychology, 7I, 1058-1067.

Di Nardo, P. A., Brown, T. A. \& Barlow, D. H. (1994) Anxiety Disorders Interview Schedule for DSM-IV Lifetime Version. Graywind.

Fedoroff, I. C. \& Taylor, S. (200I) Psychological and pharmacological treatments of social phobia: a metaanalysis. Journal of Clinical Psychopharmacology, 21, $311-324$

Fenigstein, A., Scheier, M. F. \& Buss, A. H. (1975) Public and private self-consciousness: assessment and theory. Journal of Consulting and Clinical Psychology, 43, 522-527.

Gibbons, R. D., Hedeker, D., Elkin, I., et al (1993) Some conceptual and statistical issues in analysis of longitudinal psychiatric data: application to the NIMH Treatment of Depression Collaborative Research Program dataset. Archives of General Psychiatry, 50 739-750.

Gould, R. A., Buckminster, S., Pollack, M. H., et al (1997) Cognitive-behavioral and pharmacological treatment for social phobia: a meta-analysis. Clinical Psychology: Science and Practice, 4, 291-306.

Griffiths, K. M. \& Christensen, H. (2006) Review of randomised controlled trials of Internet interventions for mental disorders and related conditions. Clinical Psychologist, 10, 16-29.

Issakidis, C. \& Andrews, G. (2002) Service utilisation for anxiety in an Australian community sample. Social Psychiatry and Psychiatric Epidemiology, 37, 153-163.

Leary, M. R. (1983) A brief version of the Fear of Negative Evaluation Scale. Personality and Social Psychology Bulletin, 9, 37I-375.
Loranger, A. W., Janca, A. \& Sartorius, N. (1997) Assessment and Diagnosis of Personality Disorders: The ICD-10 International Personality Disorder Examination (IPDE). Cambridge University Press.

Marrs, R.W. (1995) A meta-analysis of bibliotherapy studies. American Journal of Community Psychology, 23 843-870.

Mattick, R. P. \& Clarke, J. C. (1998) Development and validation of measures of social phobia scrutiny fear and social interaction anxiety. Behaviour Research and Therapy, 36, 455-470.

Meltzer, H., Bebbington, P., Brugha, T., et al (2000) The reluctance to seek treatment for neurotic disorders. Journal of Mental Health, 9, 319-327.

Newman, M. G., Erickson, T., Preworski, A., et a (2003) Self-help and minimal-contact therapies for anxiety disorders: is human contact necessary for therapeutic efficacy? Journal of Clinical Psychology, 59 $251-274$

Peters, L. (2000) Discriminant validity of the Social Phobia and Anxiety Inventory (SPAI), the Social Phobia Scale (SPS) and the Social Interaction Anxiety Scale (SIAS). Behaviour Research and Therapy, 38, 943-950.

Rapee, R. M. (1998) Overcoming Shyness and Social Phobia: A Step by Step Guide. Lifestyle Press.

Rapee, R. M. \& Sanderson, W. C. (1998) Social Phobic: Clinical Application of Evidence-Based Psychotherapy. Jason Aronson.

Rapee, R. M. \& Spence, S. H. (2004) The etiology of social phobia: empirical evidence and an initial model. Clinical Psychology Review, 24, 737-767.

Rapee, R. M., Craske, M. G. \& Barlow, D. H. (1994) Assessment instrument for panic disorder that includes fear of sensation-producing activities: the Albany Panic and Phobia Questionnaire. Anxiety, I, |14-122.

Rodebaugh, T. L., Woods, C. M., Thissen, D. M., et a (2004) More information from fewer questions: the factor structure and item properties of the original and brief Fear of Negative Evaluation Scale. Psychological Assessment, 16, 169-181.

Scogin, F., Bynum, J., Stephens, G., et al (1990) Efficacy of self-administered treatment programs: metaanalytic review. Professional Psychology: Research and Practice, 2I, 42-47.

Ware, J. E., Kosinski, M. \& Keller, S. D. (1996) A I2 item short-form health survey: construction of scales and preliminary tests of reliability and validity. Medical Care, 34, 220-233. 